\title{
Glaucoma drainage device implantation with adjunctive intravitreal bevacizumab in neovascular glaucoma: 3-year experience
}

This article was published in the following Dove Press journal:

Clinical Ophthalmology

7 August 2017

Number of times this article has been viewed

\author{
Nina Asrini Noor' \\ Syukri Mustafa ${ }^{1,2}$ \\ Widya Artini ${ }^{1,2}$ \\ 'Ophthalmology Department, \\ ${ }^{2}$ Glaucoma Division, Ophthalmology \\ Department, Cipto Mangunkusumo \\ General Hospital, Jakarta, Indonesia
}

Purpose: To evaluate the outcome of glaucoma drainage device (GDD) implantation with and without intravitreal bevacizumab (IVB) injection in treating neovascular glaucoma (NVG) at Cipto Mangunkusumo Hospital Jakarta, Indonesia.

Patients and methods: This retrospective study involved 39 eyes with NVG which underwent GDD implantation between 2012 and 2014. Thirty eyes underwent GDD implantation alone (control group) while 9 eyes underwent GDD implantation and IVB injection (IVB group). Visual acuity (VA), intraocular pressure (IOP), number of antiglaucoma medications, and success rate were compared between groups.

Results: There were no significant differences in preoperative characteristics. On the last visit, VA was $2.6(0.2-4.0) \log$ MAR in the control group and $2.3(0.4-4.0) \log$ MAR in the IVB group $(P=0.97)$. In the control group, final VA was significantly worse compared to initial VA $(P<0.01)$, while in IVB group VA was apparently stable $(P=0.24)$. Final IOP was $16.3 \pm 10.3 \mathrm{mmHg}$ in the control group and $12.0(2.0-49.0) \mathrm{mmHg}$ in IVB group $(P=0.40)$. The number of antiglaucoma medications was similar between groups $(P=0.57)$. Surgical success rate in the IVB group (66.7\%) was better than the control group (56.7\%), but this difference was not statistically significant $(P=0.71)$. Kaplan-Meier survival analysis showed the probability of success 37 months after surgery as $53.6 \%$ in the IVB group and $31.6 \%$ in the control group. No significant difference was found between the groups $(P=0.45)$.

Conclusion: In cases of NVG, GDD combined with IVB could maintain VA compared to GDD alone. However, there were no significant differences in final IOP, number of antiglaucoma medications, and surgical success rate.

Keywords: Ahmed glaucoma valve, Baerveldt implant, glaucoma surgery, vascular endothelial growth factor, VEGF

\section{Introduction}

Neovascular glaucoma (NVG) is a severe sequel of ocular ischemia due to various conditions including retinal vein occlusion, diabetic retinopathy, and ocular ischemic syndrome. ${ }^{1-3}$ In southeast Asia, a study by Shen et al in Singapore showed that $20 \%$ of 150 subjects had secondary glaucoma, and 3.3\% of those were diagnosed with NVG. ${ }^{4}$ In Indonesia, a study by Artini and Dame in 2011 at Cipto Mangunkusumo Hospital found the incidence of NVG was up to $8 \%$ between 2005 and $2007 .{ }^{5}$

NVG poses tremendous challenge in its management. It is often difficult and unpredictable to control due to its resistance toward medications and surgery. Key factors in the treatment of NVG include management of the underlying ischemic condition by eliminating angiogenic stimuli through panretinal photocoagulation followed
Correspondence: Nina Asrini Noor Ophthalmology Department, Faculty of Medicine, Universitas Indonesia, Cipto Mangunkusumo General Hospital, Jalan Kimia Nomor 8, Jakarta Pusat, 10320, Indonesia

Tel +6281395209670

Email nina.asrini.noor@gmail.com 
by intraocular pressure (IOP) control with antiglaucoma medications or surgery. ${ }^{6,7}$ Surgery is indicated in NVG with uncontrolled IOP with maximally tolerated antiglaucoma medications. Surgical modalities vary from filtering surgery with 5-fluorouracyl or mitomycin $\mathrm{C}$ as adjuvant, glaucoma drainage device (GDD) implantation, to cyclodestructive procedures. Filtering surgery and cyclodestructive procedures often fail to achieve high success rate, ${ }^{1,6,8}$ thus, GDD implantation becomes the modality of choice in NVG, especially in patients that are irresponsive to medications.

Bevacizumab (Avastin; Genentech, South San Francisco, CA, USA) is a monoclonal vascular endothelial growth factor (VEGF) inhibitor which induces regression of iris neovascularization when administered through intravitreal or intracameral route. ${ }^{9,10}$ Bevacizumab induces regression of neovascularization within 6 weeks after injection, reduces IOP, and decreases complication rate after trabeculectomy in NVG. ${ }^{4}$ Many studies have been published regarding the use of intravitreal bevacizumab (IVB) injection in conjunction with GDD implantation; however, evidence is still lacking on whether GDD implantation combined with IVB injection is beneficial for the treatment of NVG.

This retrospective study aimed to evaluate the efficacy of IVB injection with a wide range of GDD implantation in patients with NVG by comparing this procedure with GDD implantation alone in terms of visual acuity (VA), IOP, number of antiglaucoma medications, and surgical success rate.

\section{Materials and methods}

This study is a retrospective study based on a complete review of each patient's ophthalmological chart retrieved from medical records. Written informed consent according to the protocol conforming to the Declaration of Helsinki and approval from the Ethical Committee of Faculty of Medicine, Universitas Indonesia were waived. Institutional Review Board waiver was obtained and statement of patients' data confidentiality was submitted prior to the start of the study.

\section{Inclusion criteria}

This institutional retrospective study collected the medical records of patients admitted to the ophthalmology department of Cipto Mangunkusumo Kirana Hospital (Jakarta, Indonesia) diagnosed with NVG between January 2012 and December 2014. The diagnosis of NVG was made by glaucoma specialists in glaucoma clinic and defined as the presence of iris and/ or anterior chamber angle neovascularization with elevated IOP ( $\geq 21 \mathrm{mmHg}$ ). Among a total of 74 consecutive eyes with NVG, only those who underwent GDD implantation were kept. Thirty nine eyes underwent GDD implantation which included Ahmed glaucoma valve (AGV), Baerveldt implant, and Keiki Mehta implant. Exclusion criteria were age $<16$ years and follow-up duration of $<3$ months.

\section{Surgical technique}

Surgery was performed by 4 glaucoma specialists. IVB injection was administered along with GDD implantation using $1 \mathrm{cc}$ syringe and $30 \mathrm{G}$ needle through pars plana $4 \mathrm{~mm}$ posterior from the limbus into the vitreous cavity with a dosage of $1.25 \mathrm{mg} / 0.05 \mathrm{cc}$. The standard procedures of GDD implantation were as follows. Fornix-based conjunctival pocket was created followed by dissection of Tenon's capsule. Implant plate was placed and secured to the sclera at the superotemporal quadrant. Anterior chamber entrance site was created using $23 \mathrm{G}$ needles. Implant tube was inserted through the path into the anterior chamber. Scleral allograft was used to cover the tube on the sclera surface. Conjunctiva and Tenon's capsule were sutured at the limbus. After repositioning of the conjunctiva, antibiotics were injected away from the surgical site. Patients were then discharged on levofloxacin and prednisolone 6 times per day.

\section{Data collection}

Data included age at the time of diagnosis, sex, etiology of NVG, affected eye, IOP measured by Goldmann applanation tonometry or Icare ${ }^{\circledR}$ TA01i tonometer, VA presented in $\log$ MAR, number of antiglaucoma medications used, follow-up duration, and intraoperative IVB injection. Measurements of IOP were taken preoperatively and postoperatively at 1 month, 3 months, and at the last follow-up. VA was measured preoperatively and postoperatively at the final visit. VA of counting fingers was equivalent to $2.3 \log \mathrm{MAR}$, hand movement was equivalent to $2.6 \log$ MAR, light perception (LP) was $3.0 \operatorname{logMAR}$, and no light perception (NLP) was 4.0 logMAR. ${ }^{11}$ All patients were under maximally tolerated antiglaucoma medications before surgery, except in cases of major contraindications. Surgical success was defined as stabilization of IOP between 6 and $21 \mathrm{mmHg}$ with or without antiglaucoma medications, without the loss of LP. Surgical failure was defined as IOP $<6 \mathrm{mmHg}$ or $>21 \mathrm{mmHg}$, deterioration of VA toward NLP, or the need for additional glaucoma surgeries.

\section{Statistical analysis}

Statistical analysis and graphics were performed using IBM SPSS 20.0 (IBM Corporation, Armonk, NY, USA). The results were presented as mean $\pm \mathrm{SD}$ or median (range) according 
to the distribution of the data, and percentages. Qualitative data were compared using chi-square or Fisher's exact test, and quantitative data using independent-samples $t$-test or Mann-Whitney test and paired-samples $t$-test or Wilcoxon test according to the distribution of data. The cumulative probability of surgical success was analyzed by Kaplan-Meier method and log-rank test was used to compare the calculated success rate. Statistical significance was defined as $P<0.05$.

\section{Results}

There were 44 cases from 41 patients with NVG who underwent GDD implantation between January 2012 and December 2014. Five patients had a follow-up period of $<3$ months; thus, they were excluded from the study. Finally, a total of 39 eyes from 36 patients were analyzed.

Out of 39 eyes, 30 eyes underwent GDD implantation only (control group) while 9 eyes underwent GDD implantation in combination with IVB injection (IVB group). Table 1 represents the demographic and baseline characteristics of both groups. No significant differences were observed in terms of age, gender distribution, involved eye, etiology, and follow-up period. Both groups also shared a similar proportion regarding type of implants, in which AGV implant was the most commonly used. VA, IOP, and number of antiglaucoma medications were also not significantly different between the two groups.

Comparison of VA, IOP, and number of medications at the final postoperative visit between groups is shown in Table 2 .

Table I Baseline demographic and clinical characteristics of the two study groups

\begin{tabular}{|c|c|c|c|}
\hline & $\begin{array}{l}\text { Control } \\
\text { group }(n=30)\end{array}$ & $\begin{array}{l}\text { IVB group } \\
(n=9)\end{array}$ & $P$-value \\
\hline Age (years) & $55.5 \pm 15.8$ & $52.5 \pm 16.3$ & $0.64^{\mathrm{a}}$ \\
\hline \multicolumn{4}{|l|}{ Sex, n (\%) } \\
\hline Male & $16(53.3 \%)$ & $4(44.4 \%)$ & $0.72^{b}$ \\
\hline Female & 14 (46.7\%) & $5(55.6 \%)$ & \\
\hline \multicolumn{4}{|l|}{ Etiology, n (\%) } \\
\hline Diabetic retinopathy & 17 (56.7\%) & $6(66.7 \%)$ & $1.00^{\mathrm{b}}$ \\
\hline Retinal vein occlusion & $4(13.3 \%)$ & I (II.I\%) & \\
\hline Others & $9(30.0 \%)$ & $2(22.2 \%)$ & \\
\hline \multicolumn{4}{|l|}{ Implant type, n (\%) } \\
\hline Ahmed glaucoma valve & $13(43.3 \%)$ & $5(55.6 \%)$ & $0.89^{b}$ \\
\hline Baerveldt implant & $10(33.3 \%)$ & $2(22.2 \%)$ & \\
\hline Keiki Mehta implant & 7 (23.3\%) & $2(22.2 \%)$ & \\
\hline Follow-up period (months) & $9.0(4.0-37.0)$ & $15.0(4.0-37.0)$ & $0.33^{c}$ \\
\hline Initial visual acuity (logMAR) & $2.3(0.2-3.0)$ & $2.3(0.5-3.0)$ & $0.74^{c}$ \\
\hline Initial IOP (mmHg) & $49.3 \pm 14.7$ & $45.1 \pm 13.0$ & $0.42^{\mathrm{a}}$ \\
\hline Initial number of medications & $2.0(2.0-3.0)$ & $2.0(2.0-3.0)$ & $0.43^{c}$ \\
\hline
\end{tabular}

Notes: Data presented as mean \pm standard deviation or median (min-max) according to the distribution of the data. 'Student's $t$-test; 'Fisher's exact test; Mann-Whitney test.

Abbreviations: IVB, intravitreal bevacizumab; IOP, intraocular pressure; SD, standard deviation.
Table 2 Comparison of final postoperative clinical conditions between the two groups

\begin{tabular}{llll}
\hline & $\begin{array}{l}\text { Control } \\
\text { group }(\mathbf{n}=\mathbf{3 0})\end{array}$ & $\begin{array}{l}\text { IVB group } \\
(\mathbf{n}=\mathbf{9})\end{array}$ & P-value \\
\hline Final visual acuity (logMAR) & $2.6(0.2-4.0)$ & $2.3(0.4-4.0)$ & $0.97^{\mathrm{a}}$ \\
Final IOP (mmHg) & $16.3 \pm 10.3$ & $12.0(2.0-49.0)$ & $0.40^{\mathrm{a}}$ \\
$\begin{array}{l}\text { Final number of medications } \\
\text { Success rate, } \mathrm{n}(\%)\end{array}$ & $1.0(0-4.0)$ & $1.0(0-2.0)$ & $0.57^{\mathrm{a}}$ \\
$\quad \begin{array}{l}\text { Success } \\
\text { Failure }\end{array}$ & $17(56.7 \%)$ & $6(66.7 \%)$ & $0.7 \mathrm{I}^{\mathrm{b}}$ \\
\hline
\end{tabular}

Notes: Data presented as mean \pm standard deviation or median (min-max) according to the distribution of the data. ${ }^{\text {Mann-Whitney test; }}$ 'Fisher's exact test.

Abbreviations: IVB, intravitreal bevacizumab; IOP, intraocular pressure.

All three variables showed no significant difference between the control group and IVB group. In terms of surgical success rate, IVB group had a higher success rate compared to the control group, $66.7 \%$ and $56.7 \%$, respectively. However, this difference was not proven to be significant $(P=0.71)$. Within group comparison between preoperative and postoperative conditions of all variables can be seen in Table 3. Control group showed significant decrease in the final VA which was 2.3-2.6 logMAR $(P<0.01)$, whereas IVB group showed unchanged VA at $2.3 \log$ MAR $(P=0.24)$. Substantial changes of IOP and number of medications were observed in both groups.

IOP distribution at different follow-up times is shown in Figure 1. Major decrease of IOP to $<21 \mathrm{mmHg}$ was seen as early as 1 month postoperatively in both IVB and control groups. The IVB group tended to have lower IOP compared to the control group at 1 and 3 months of follow-up, although the differences were not proven to be statistically significant. IOP changes from 1 month to final follow-up also showed a tendency to be lower in both groups despite it being statistically insignificant.

In terms of GDD type, additional analysis was performed between all GDD implant types. There were no statistically significant differences between AGV implant, Baerveldt implant, and Keiki Mehta implant in terms of 1 month, 3 months, and final postoperative IOP $(P$-value $=0.70,0.19$, and 0.78 , respectively).

A comparison of Kaplan-Meier survival curve analysis between the two groups is presented in Figure 2. The probability of success 37 months after surgery was $53.6 \%$ in the IVB group and $31.6 \%$ in the control group. The log-rank test revealed no significant difference between the groups $(P=0.45)$.

\section{Discussion}

Ischemia in more than half the retina causes tissue hypoxia which leads to expression and release of angiogenic factors, as seen in NVG. VEGF is one of major angiogenic 
Table 3 Comparison of preoperative and postoperative clinical conditions within each group

\begin{tabular}{|c|c|c|c|c|c|c|}
\hline & \multicolumn{3}{|c|}{ Control group $(n=30)$} & \multicolumn{3}{|c|}{ IVB group $(n=9)$} \\
\hline & Initial & Final & $P$-value & Initial & Final & $P$-value \\
\hline Final visual acuity (logMAR) & $2.3(0.2-3.0)$ & $2.6(0.2-4.0)$ & $<0.0 \mathrm{I}^{\mathrm{a}}$ & $2.3(0.5-3.0)$ & $2.3(0.4-4.0)$ & $0.24^{\mathrm{a}}$ \\
\hline Final IOP $(\mathrm{mmHg})$ & $49.3 \pm 14.7$ & $16.3 \pm 10.3$ & $<0.0 \mathrm{I}^{\mathrm{b}}$ & $45.1 \pm 13.0$ & $12.0(2.0-49.0)$ & $0.0 \mathrm{I}^{\mathrm{a}}$ \\
\hline Final number of medications & $2.0(2.0-3.0)$ & $1.0(0-4.0)$ & $<0.0 \mathrm{I}^{\mathrm{a}}$ & $2.0(2.0-3.0)$ & $1.0(0-2.0)$ & $<0.0 \mathbf{I}^{\mathrm{a}}$ \\
\hline
\end{tabular}

Notes: Data presented as mean \pm standard deviation or median (min-max) according to the distribution of the data. Data in bold indicates statistically significant difference between the two conditions (initial and final) of each group (control and IVB group). ${ }^{a}$ Wilcoxon test; ${ }^{b}$ paired $t$-test.

Abbreviations: IVB, intravitreal bevacizumab; IOP, intraocular pressure.

factors involved. Accumulation of VEGF along with other angiogenic factors induces formation of iris neovascularization and anterior synechia leading to increased IOP.,2,7

GDD implantation becomes the first-line treatment alternative in NVG management because of its effectiveness and low postoperative complication rate. ${ }^{2}$ Although it is considered effective, NVG itself is a risk factor toward failure after GDD implantation. ${ }^{12}$ Currently, the choice between available implants (Ahmed, Baerveldt, etc) depends on surgeon's preference and experience without affecting final outcomes and complications. ${ }^{13}$

Recently, the use of anti-VEGF agents for the treatment of NVG has been found to be beneficial. Although its effectiveness is proven to be limited in a short period of time, administration of bevacizumab is proven to show benefits. ${ }^{14}$

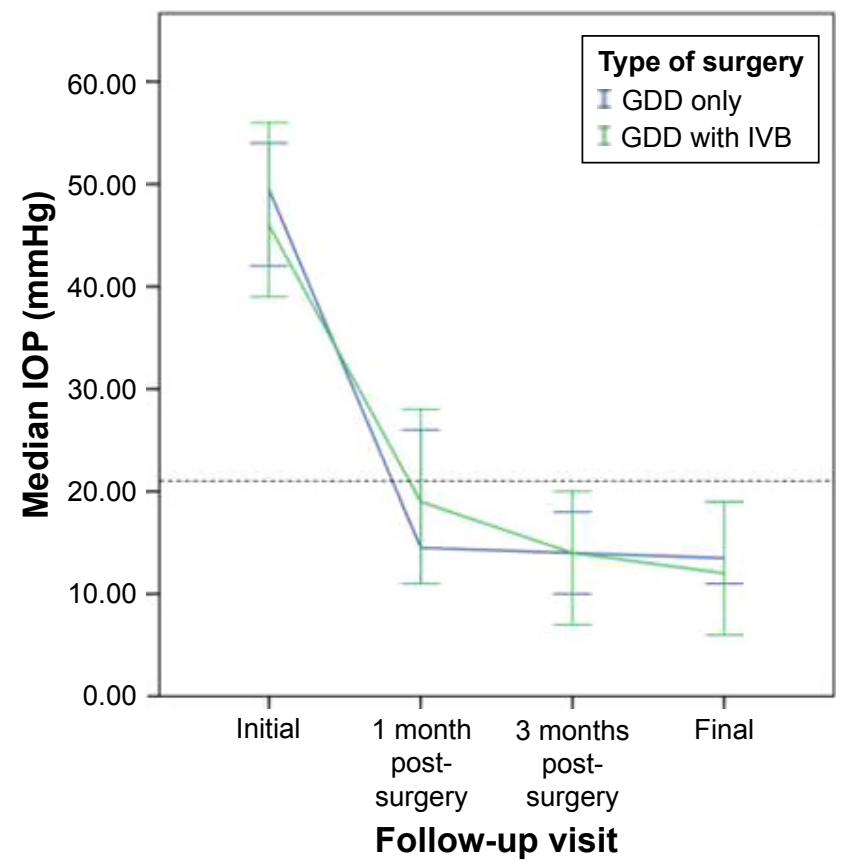

Figure I IOP distribution in control group (30 eyes, blue line) and IVB group ( 9 eyes, green line) at various follow-up periods.

Notes: IOP decreased at all time points compared with preoperative IOP in both groups. However, the differences of IOP between the groups were not statistically significant at any period. Error bars: $95 \% \mathrm{Cl}$.

Abbreviations: IVB, intravitreal bevacizumab; IOP, intraocular pressure; GDD, glaucoma drainage device; $\mathrm{Cl}$, confidence interval.
Besides, bevacizumab can be used in combination with GDD implantation in patients with NVG. ${ }^{15}$

The purpose of this study was to evaluate the outcomes of GDD implantation with and without the combination of IVB injection regarding VA, IOP, and number of antiglaucoma medications. Additionally, the potential of IVB injection toward surgical success rate was also assessed.

Bevacizumab seemed to have little effect on visual prognosis in eyes with NVG. In this study, no significant difference was observed regarding the final VA of both IVB group and control group. This finding was similar to other studies which stated that bevacizumab was not beneficial in terms of visual outcome. ${ }^{7,16}$ In this study, loss of LP was observed in $22.2 \%$ of cases in IVB group compared to $26.7 \%$ in the control group. Similar result was observed in a study by Sahyoun et al in which the rate of loss of LP was lower in IVB group. ${ }^{7}$ Although IVB did not have the ability to improve

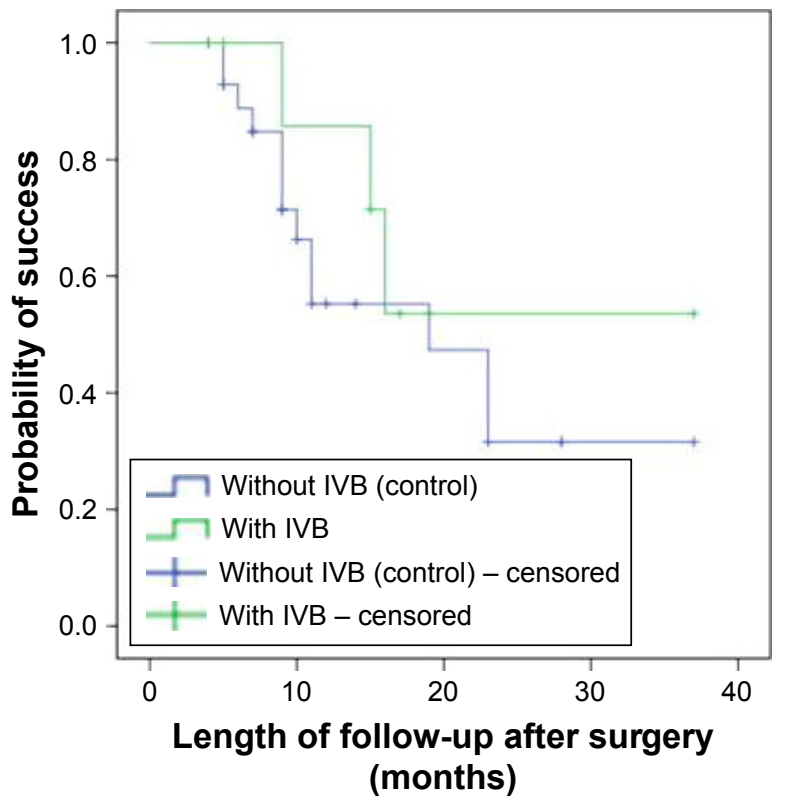

Figure 2 Cumulative probability of success after surgery in IVB group ( 9 eyes, green line) and control group ( 30 eyes, blue line) using Kaplan-Meier survival curve analysis.

Note: Log-rank test revealed no significant difference in the survival time between groups.

Abbreviation: IVB, intravitreal bevacizumab. 
vision, it seemed to be able to maintain VA. It is also shown in our study that the control group suffered significant visual deterioration while the IVB group did not at the final visit. A study by Kang et al showed that IVB might provide better VA up to 1 month postoperatively by reducing the rate of hyphema and macular edema. ${ }^{17}$

GDD implantation was effective in decreasing the IOP as seen in this study in which all cases had lower IOP until last follow-up. Final IOP was different between 3 types of GDD implants. IOP in the IVB group was slightly lower than that in the control group, $12.0(2.0-49.0)$ and $16.3 \pm 10.3 \mathrm{mmHg}$, respectively. Recent studies reported that IVB injection, whether given intraoperatively or preoperatively, may contribute to reducing the IOP after surgery. ${ }^{15,18}$ IVB injection given as adjunctive treatment in recalcitrant NVG may affect the reversible part of synechia in anterior chamber angle and further reduce the IOP after GDD implantation. ${ }^{17}$

Surgical success rate in the IVB group was higher than the control group, though not statistically significant. Similar results were seen in other studies in which IVB resulted in a higher success rate although not proven significant. ${ }^{17,19}$ The transient nature of IVB might contribute to a lack of the improved probability in success, as postulated by Takihara et al. ${ }^{18}$ Regression of iris neovascularization persists for 8-10 weeks after IVB injection but then returns to its previous condition within 6 months after administration. ${ }^{20,21}$ The accumulation and elevation of VEGF levels in aqueous humor and vitreous may further induce tissue fibrosis around the place which then leads to surgical failure. ${ }^{19}$

This study had several limitations. First, selection bias could not be avoided completely due to its retrospective nature. To address this bias, we compared the preoperative characteristics of both groups and found no significant differences. Second, the minimum of 3 months follow-up duration may be inadequate to obtain the true effect of IVB injection. However, the number of patients with a longer follow-up duration were too few to acquire statistical results. Further prospective and randomized studies with long-term follow-up would provide clearer results of the effect of intravitreal antiVEGF in patients with NVG. Third, the lack of a standardized protocol of IVB injection in NVG especially regarding timing of administration may also contribute as a limitation in this study. The last and a major limitation to this study was the unbalanced number of subjects between the groups. Due to the design limitation of the study, we were unable to have a comparable number of subjects in each group. Therefore, it is rather difficult to draw a direct conclusion and it should be understood carefully.

\section{Conclusion}

GDD implantation provided good outcomes in NVG management in terms of IOP control and reduction of antiglaucoma medications. GDD implantation combined with IVB injection might offer the potential in maintaining VA compared to GDD alone despite IOP control and number of medications remaining the same. Prospective study with a larger sample size and longer period of follow-up is warranted. IVB injection protocol should also be standardized to confirm efficacy and safety of this treatment modality.

\section{Disclosure}

The authors report no conflicts of interest in this work.

\section{References}

1. Chen C, Lai I, Wu P, et al. Adjunctive intravitreal bevacizumabcombined trabeculectomy versus trabeculectomy alone in treatment of neovascular glaucoma. J Ocul Pharmacol Ther. 2010;28(1):111-118.

2. Hayreh SS. Neovascular glaucoma. Prog Retin Eye Res. 2007;26: 470-485.

3. Kiddee W, Tantisarasart T, Wangsupadilok B. Neovascular glaucoma: a retrospective review of 5-year experience in Songklanagarind Hospital. J Med Assoc Thai. 2012;95(Suppl 4):S36-S42.

4. Shen SY, Wong TY, Foster PJ, et al. The prevalence and types of glaucoma in Malay people: the Singapore Malay eye study. Invest Ophthalmol Vis Sci. 2008;49:3846-3851.

5. Artini W, Dame. Glaucoma caused blindness with its characteristic in Cipto Mangunkusumo Hospital. Jurnal Oftalmologi Indonesia. 2011;7(5):189-193. Indonesian.

6. Alkawas AA, Shahien EA, Hussein AM. Management of neovascular glaucoma with panretinal photocoagulation, intravitreal bevacizumab, and subsequent trabeculectomy with mitomycin C. J Glaucoma. 2010;19(9):622-626.

7. Sahyoun M, Azar G, Khoueir Z, et al. Long-term results of Ahmed glaucoma valve in association with intravitreal bevacizumab in neovascular glaucoma. J Glaucoma. 2015;24(5):383-388.

8. Takihara Y, Inatani M, Fukushima M, et al. Trabeculectomy with mitomycin C for neovascular glaucoma: prognostic factors for surgical failure. Am J Ophthalmol. 2009;147:911-918.

9. Chilov MN, Grigg JR, Playfair TJ. Bevacizumab (Avastin) for the treatment of neovascular glaucoma. Clin Exp Ophthalmol. 2007;35: 494-496.

10. Costagliola $\mathrm{C}$, Cipollone U, Rinaldi $\mathrm{M}$, et al. Intravitreal bevacizumab (Avastin) injection for neovascular glaucoma: a survey on 23 cases throughout 12-month follow-up. Br J Clin Pharmacol. 2008;66: 667-673.

11. Scott IU, Schein OD, West S. Functional status and quality of life measurement among ophthalmic patients. Arch Ophthalmol. 1994; 112:329-335.

12. Netland PA, Ishida K, Boyle JW. The Ahmed glaucoma valve in patients with and without neovascular glaucoma. J Glaucoma. 2010;19: 581-586.

13. Christakis PG, Tsai JC, Zurakowski D, et al. The Ahmed versus Baerveldt study: design, baseline patient characteristics, and intraoperative complications. Ophthalmology. 2011;118:2172-2179.

14. Iliev ME, Domig D, Wolf-Schnurrbursch U, et al. Intravitreal bevacizumab (Avastin) in the treatment of neovascular glaucoma. Am J Ophthalmol. 2006;142:1054-1056.

15. Ma KT, Yang JY, Kim NR, et al. Surgical results of Ahmed valve implantation with intraoperative bevacizumab injection in patients with neovascular glaucoma. J Glaucoma. 2012;21:331-336. 
16. Yazdani S, Hendi K, Pakravan M, et al. Intravitreal bevacizumab for neovascular glaucoma: a randomized controlled trial. J Glaucoma. 2009;18:632-637.

17. Kang JY, Nam KY, Lee SJ, et al. The effect of intravitreal bevacizumab injection before Ahmed valve implantation in patients with neovascular glaucoma. Int Ophthalmol. 2014;34(4):793-799.

18. Takihara Y, Inatani M, Kawaji T, et al. Combined intravitreal bevacizumab and trabeculectomy with mitomycin $\mathrm{C}$ versus trabeculectomy with mitomycin C alone for neovascular glaucoma. J Glaucoma. 2011; 20:196-201.
19. Sevim MS, Buttanri IB, Kugu S, et al. Effect of intravitreal bevacizumab injection before Ahmed glaucoma valve implantation in neovascular glaucoma. Ophthalmologica. 2013;229:94-100.

20. Silva Paula J, Jorge R, Alves Costa R, et al. Short-term results of intravitreal bevacizumab (Avastin) on anterior segment neovascularization in neovascular glaucoma. Acta Ophthalmol Scand. 2006;84:5556-5557.

21. Wakabayashi T, Oshima Y, Sakaguchi H, et al. Intravitreal bevacizumab to treat iris neovascularization and neovascular glaucoma secondary to ischemic retinal diseases in 41 consecutive cases. Ophthalmology. 2008;115:1571-1580
Clinical Ophthalmology

\section{Publish your work in this journal}

Clinical Ophthalmology is an international, peer-reviewed journal covering all subspecialties within ophthalmology. Key topics include: Optometry; Visual science; Pharmacology and drug therapy in eye diseases; Basic Sciences; Primary and Secondary eye care; Patient Safety and Quality of Care Improvements. This journal is indexed on

\section{Dovepress}

PubMed Central and CAS, and is the official journal of The Society of Clinical Ophthalmology (SCO). The manuscript management system is completely online and includes a very quick and fair peer-review system, which is all easy to use. Visit http://www.dovepress.com/ testimonials.php to read real quotes from published authors. 